\title{
Uso de escorias de alto horno y acero en mezclas asfálticas: revisión ${ }^{*}$
}

\author{
Hugo Alexander Rondón Quintana* \\ Marcio Muniz de Farias ${ }^{* * *}$ \\ Fredy Alberto Reyes Lizcano ${ }^{* * *}$
}

\author{
Recibido: 02/02/2017 • Aceptado: 04/03/2018 \\ https://doi.org/10.22395/rium.v17n33a4
}

\begin{abstract}
Resumen
En la fabricación de mezclas asfálticas se consumen grandes cantidades de agregados pétreos de origen natural lo que impacta negativamente el ambiente. Estos agregados pueden ser sustituidos parcial o totalmente por otros que son desechados en escombreras y producidos por las compañías siderúrgicas, como las escorias de alto horno (BFS) y acero (SS). Estos dos últimos materiales poseen propiedades químicas y físicas que los hacen aptos para ser utilizados en múltiples aplicaciones como la construcción, mantenimiento y rehabilitación de carreteras. En el artículo se presenta una revisión del estado del conocimiento del empleo de BFS y SS en la producción de mezclas asfálticas, se describen y definen ambos materiales, se presenta la problemática ambiental, su riesgo toxicológico de uso, sus propiedades químicas y físicas, las ventajas y limitaciones de uso y la forma como han sido estudiados para ser empleados como agregados pétreos de mezclas asfálticas. Con base en la revisión bibliográfica realizada, los autores al final del artículo exponen algunas recomendaciones para continuar los estudios tendientes a sustituir agregados pétreos naturales por BFS y SS en la fabricación de mezclas asfálticas.
\end{abstract}

Palabras clave: escoria de alto horno; escoria de acero; mezclas asfálticas; medioambiente.

* Artículo de revisión derivado del proyecto de investigación posdoctoral Comportamiento de una mezcla asfáltica tibia fabricada con escorias de acero y alto horno financiado por la Universidad Distrital "Francisco José de Caldas", por la Universidade de Brasília y por la Pontificia Universidad Javeriana.

** Doctor en Ingeniería. Universidad Distrital “Francisco José de Caldas". Facultad del Medio Ambiente y Recursos Naturales. Grupo de investigación Centro de Estudios en Pavimentos y Materiales Sostenibles. Carrera 5 Este N. 15-82, sede El Vivero, Teléfono: (+57) 310 8687715. Correo electrónico: harondonq@udistrital.edu.co.

*** Doctor of Philosophy. Universidade de Brasília. Faculdade de Tecnologia, Campus Universitário Darcy Ribeiro, Brasília _CEP 70910-900. Teléfono: (+55) 61 99970-5484. Correo electrónico: muniz@unb.br. Orcid: https:// orcid.org/0000-0002-5257-911X

${ }^{* * * *} \mathrm{Ph}$. D. en Ingeniería. Pontificia Universidad Javeriana. Grupo de investigación Cecata. Calle 40 carrera 7 ${ }^{\mathrm{a}}$, Bogotá, Colombia. Teléfono (+57) 13208320 ext.5270. Correo electrónico: fredy.reyes@javeriana.edu.co. Orcid: https:// orcid.org/0000-0002-6407-168X 


\title{
Use of Blast Furnace Slag and Steel in Asphalt Mixtures: Review
}

\begin{abstract}
In the manufacture of asphalt mixtures, large quantities of natural stone aggregates are consumed, which has a negative impact on the environment. These aggregates can be partially or totally replaced by others that are disposed of in dumps and produced by steel companies, such as blast furnace slag (BFS) and steel (SS). These last two materials have chemical and physical properties that make them suitable for use in multiple applications such as road construction, maintenance and rehabilitation. The article presents a review of the state of knowledge of the use of BFS and SS in the production of asphalt mixtures, describes and defines both materials, presents the environmental problems, their toxicological risk of use, their chemical and physical properties, the advantages and limitations of use and the way they have been studied to be used as stone aggregates of asphalt mixtures. Based on the bibliographic review, the authors at the end of the article present some recommendations to continue the studies tending to substitute natural stone aggregates with BFS and SS in the manufacture of asphalt mixtures.
\end{abstract}

Keywords: blast furnace slag; steel slag; asphalt mixtures; environment.

\section{Uso de escórias de alto-forno e aço em misturas asfálticas: revisão}

\begin{abstract}
Resumo
Grandes quantidades de agregados pétreos de origem natural são consumidos na fabricação de misturas asfálticas, o que impacta negativamente o ambiente. Esses agregados podem ser substituídos parcial ou totalmente por outros que são descartados em aterros e produzidos pelas companhias siderúrgicas, como as escórias de alto-forno (BFS) e aço (SS). Esses dois últimos materiais apresentam propriedades químicas e físicas que os tornam aptos para serem utilizados em múltiplas aplicações, como a construção, a manutenção e a reabilitação de estradas. No artigo, apresenta-se uma revisão do estado do conhecimento do emprego de BFS e SS na produção de misturas asfálticas, ambos os materiais são descritos e definidos, apresenta-se a problemática ambiental, seu risco toxicológico de uso, suas propriedades químicas e físicas, as vantagens e limitações de uso e a forma como foram estudados para serem empregados como agregados pétreos de misturas asfálticas. Com base na revisão bibliográfica realizada, os autores ao final do artigo expõem algumas recomendações para continuar os estudos com tendência a substituir agregados pétreos naturais por BFS e SS na fabricação de misturas asfálticas.
\end{abstract}

Palavras-chave: escória de alto-forno; escoria de aço; misturas asfálticas; meio ambiente. 


\section{INTRODUCCIÓN}

\section{Planteamiento del problema y motivación}

Por lo general, los agregados pétreos conforman más del $90 \%$ y del $75 \%$ de la masa y del volumen de mezclas asfálticas, respectivamente. Esto hace que, en la construcción, mantenimiento y rehabilitación de vías, se consuman grandes cantidades de este material, el cual es principalmente de origen natural. Lo anterior redunda en un impacto ambiental negativo. En las últimas dos décadas ha venido creciendo el interés por reemplazar materiales granulares naturales (MGN) por otros alternativos, con el objetivo de conservar los recursos naturales, reducir el espacio que ocupan al final de su vida útil en las escombreras e impedir el deterioro del paisaje [1]. Uno de los materiales alternativos que pueden ser utilizados como sustitutos de MGN son las escorias de alto horno (BFS, por sus siglas en inglés) y las de acero (SS, por sus siglas en inglés). Importantes cantidades de estos materiales se generan como desecho diariamente en las industrias de acero. Para dar un ejemplo, de acuerdo con [2], en EE.UU. se producen aproximadamente ocho millones de toneladas de escoria de acero por año, mientras que en Europa se producen doce millones de toneladas [3]. El método convencional de eliminar escorias es llevándolas a escombreras. La creciente eliminación de escorias no solo ocupa espacios de terreno, sino que también desperdicia recursos que pueden ser reutilizados y que, potencialmente, pueden tener un impacto en el medio ambiente debido a la contaminación del agua, el aire y el suelo producto de la liberación de metales y elementos químicos que pueden causar problemas ambientales [4]. Desde el punto de vista ambiental, entonces, la sustitución de MGN por escorias es una alternativa interesante de estudio en el área de las mezclas asfálticas. Incluso las escorias pueden ser una alternativa técnica y económica en zonas donde escasean MGN de buenas propiedades físicas y donde es necesario traer dichos materiales de regiones distantes con el consecuente encarecimiento del costo de los proyectos viales [5]. Por otro lado, con respecto al uso de materiales alternativos, aún existen diversas inquietudes ligadas a la evaluación del desempeño técnico y ambiental en proyectos viales, las cuales no han sido respondidas de manera satisfactoria [6].

Diversos estudios han sido ejecutados en el mundo con el fin de evaluar las propiedades de las escorias para diferentes aplicaciones. En el presente estudio se realiza una revisión bibliográfica sobre el estado actual del conocimiento y se describen las diversas investigaciones que han sido ejecutadas sobre escorias (BFS y SS), con el fin de evaluar su uso potencial en la fabricación de mezclas asfálticas. De la bibliografía consultada no existe actualmente una revisión que profundice sobre el uso de escorias para producir mezclas asfálticas y, en ese sentido, el presente artículo hace un aporte. El objetivo principal del estudio es tomar las conclusiones del mismo como punto de 
partida para la ejecución de un proyecto de investigación que se está realizando en la Universidad de Brasilia sobre el desarrollo de una mezcla asfáltica tibia reciclada bajo parámetros técnicos, económicos y medioambientales. Asimismo, la presente revisión bibliográfica sirve como punto de partida para que otros investigadores y grupos de investigación la consulten y tengan en cuenta en futuros estudios sobre el tema.

\section{Riesgo toxicológico de uso de BFS y SS}

La liberación de metales o elementos nocivos de las escorias puede causar problemas ambientales como la contaminación del agua y del suelo, y un riesgo toxicológico para los seres humanos a través de la inhalación de pequeñas partículas $(<10 \mu \mathrm{m})$. Sin embargo, de acuerdo con [2] y [7], las escorias BFS y SS no deben considerarse como un residuo peligroso, ya que no superan la concentración de toxicidad especificada como peligrosa de acuerdo al criterio TCLP (Toxicity Characteristic Leaching Procedure) [8], En estos estudios se realizaron ensayos de lixiviación de metales pesados empleando las metodologías ICPAES (Inductively Coupled Plasma-Atomic Emission Spectrometer) y TCLP. Se concluyó que ambos materiales están dentro de los límites legales de emisiones contaminantes en Italia. En un estudio realizado por [9], se encontró que las SS no representan ninguna amenaza para los seres humanos, los animales o el medio ambiente. De acuerdo con [3], las carreteras construidas con SS, ya sea que se empleen como materiales granulares no tratados o ligados, no influyen en el medio ambiente por lixiviación. Por su parte [10], con base en un análisis químico y empleando un espectrofotómetro de absorción atómica de grafito para evaluar la toxicidad del metal lixiviado de SS, concluyen que los materiales estudiados pueden utilizarse de manera segura en la construcción de carreteras. De otro lado, Barišić et al. [11], realizaron ensayos para evaluar el efecto, sobre gusanos, de la composición química, el oxígeno disuelto, la radioactividad, los biomarcadores moleculares y los valores de $\mathrm{pH}$ del lixiviado de dos SS y concluyeron que no representan un riesgo para el medio ambiente cuando son usadas en proyectos de carreteras. Sin embargo, reportan que la cantidad de vanadio debe ser monitoreado en dichos proyectos. Según [12], la lixiviación de metales pesados en escorias es baja y no son caracterizados como materiales tóxicos (teniendo como base las especificaciones internacionales y griegas). Por su parte [13], basado en ensayos de lixiviación sobre SS confirma que los metales pesados de la SS no se pueden separar fácilmente, incluso en condiciones ácidas. En [14] fueron realizadas pruebas de lixiviación sobre cinco mezclas asfálticas fabricadas con agregado compuesto por $40 \%$ de SS fabricadas en arco eléctrico. Ellos reportan que no puede considerarse insignificante la cantidad de lixiviado medido, sin embargo, resultó aceptable. Según [15], en proyectos de carreteras, las escorias pueden causar liberación de cromo y vanadio que son elementos tóxicos. Basados en ensayos de lixiviación, reportaron que la liberación de cromo es baja mientras que la de vanadio puede ser 
importante. En [16] también reportaron que, en mezclas asfálticas compuestas por SS, la lixiviación de cromo es baja y no afecta al medio ambiente. Adicionalmente [17] y [18] concluyen que las escorias ayudan a incrementar el $\mathrm{pH}$ de aguas subterráneas. Asimismo, ayudan a aumentar el pH en los suelos [19]. Una conclusión similar con respecto al agua fue reportada por [20] en donde se menciona que el $\mathrm{pH}$ alto y el contenido de aluminio pueden lixiviar a niveles que pueden ser dañinos para la vida acuática. Sin embargo, estos últimos autores mencionan, que ninguna de las aplicaciones actuales de la escoria de la industria siderúrgica plantea un riesgo potencialmente significativo para la salud humana o una amenaza para las fuentes de agua potable de las aguas subterráneas.

\section{ESCORIAS DE ALTO HORNO}

\subsection{Generalidades}

Las escorias pueden ser clasificadas en tres categorías: las ferrosas, como las escorias de alto horno (BFS por sus siglas en inglés), las no ferrosas y las que se producen por incineración [4]. Las BFS se generan durante el proceso de obtención de hierro. Se forman cuando el mineral de hierro, coque y un fundente (ya sea de piedra caliza o dolomita) se funden juntos en un alto horno. Cuando el proceso de fundición metalúrgico se completa, la cal en el flujo se ha combinado químicamente con los aluminatos y silicatos de la ceniza de mineral y el coque, formando la escoria de alto horno [21, 22]. Las escorias al salir de los altos hornos son enfriadas lentamente al aire libre (ACBFS, Air-Cooled Blast Furnace Slag) o rápidamente aplicándoles chorros de agua fría (escorias expandidas) [23]. Las primeras son utilizadas principalmente en la construcción como agregado pétreo (concreto y materiales de relleno, entre otros), mientras las segundas son más utilizadas en la producción de cemento. Asimismo, las escorias expandidas, debido a su mayor porosidad y reducido peso en comparación con las ACBFS [24], son utilizadas en la fabricación de concretos y mampuestos ligeros. Las BFS contienen poco hierro $(\mathrm{FE}<2 \%$ ) en comparación con las de acero (SS) [4].

De acuerdo con [2] y [25], la producción anual de BFS en Japón y Reino Unido es de 24,3 y 4,0 millones de toneladas, respectivamente. Según [2, 26, 27], por cada tonelada de hierro producido se genera una cantidad de BFS entre 220 y $370 \mathrm{~kg}, 340$ y $421 \mathrm{~kg}$ y 260 y $300 \mathrm{~kg}$, respectivamente. De acuerdo con [27], la producción de BFS representa casi el $30 \%$ del calor residual liberado por las industrias manufactureras de hierro y acero. Hace tres décadas, más de 13 millones de toneladas de BFS eran producidas en EE. UU. [28].

El método convencional para eliminar escorias es transportándolas y depositándolas en escombreras. Lo anterior genera un problema ambiental, ya que ocupan 
espacios dentro de los rellenos sanitarios y se desperdicia un material que puede ser reutilizado. Según [6], al utilizar BFS se podría i) reducir el uso de agregados pétreos naturales, evitando así, adicionalmente la liberación de contaminantes en el aire, el agua y el suelo durante el procesamiento de dichos materiales, ii) disminuir el consumo de energía durante el proceso de extracción de agregados naturales.

Las BFS han sido utilizadas ampliamente en el mundo desde hace más de un siglo. Algunos usos han sido: como fertilizantes [7], en la construcción de carreteras [29], en la recuperación de suelos y en la preparación de materiales tales como vidrio de cerámica, gel de sílice, ladrillos, entre otros [26]. Sin embargo, tal vez su mayor uso es en la producción de cemento [14, 26, 28, 30, 31], ya que este tipo de escoria [29]: i) contiene poco hierro, alto contenido de óxido de calcio y composición similar al clinker, ii) es un material que puede ser utilizado de forma segura junto con el clinker y el yeso, iii) debido al bajo contenido de hierro que contienen pueden ser usadas con seguridad en la producción de cemento, iv) ayudan a desarrollar en el cemento propiedades tales como bajo calor de hidratación, buena resistencia a largo plazo y a los ácidos, v) permiten hacer ahorros a las industrias del cemento. Adicional a lo anterior, [30] y [32] mencionan que las BFS exhiben propiedades cementantes. Con respecto al uso de BFS en proyectos de construcción, existen pocas preocupaciones ambientales, ya que los metales y componentes en dichas escorias están fuertemente adheridos a su matriz y no lixivian fácilmente.

\subsection{Propiedades de las BFS y uso en mezclas asfálticas}

En la tabla 1 se presenta la composición química típica de las BFS.

Tabla 1. Composición química de BFS en \%.

\begin{tabular}{|l|c|c|c|c|c|c|c|c|c|}
\hline Componente & {$[33]$} & {$[34]$} & {$[26]$} & {$[35]$} & {$[29]$} & {$[36]$} & {$[32]$} & {$[37]$} & {$[38]$} \\
\hline Óxido de calcio $(\mathrm{CaO})$ & $36-45$ & 40 & 37,4 & 43,3 & 46,98 & 36,44 & 40,35 & 20,83 & 40,37 \\
\hline Óxido de silicio $\left(\mathrm{SiO}_{2}\right)$ & $33-42$ & 36,8 & 37,14 & 25,5 & 16,18 & 31,76 & 33,26 & 52,03 & 37,47 \\
\hline Óxido de aluminio $\left(\mathrm{Al}_{2} \mathrm{O}_{3}\right)$ & $10-16$ & 16 & 9,15 & 17 & 6,63 & 14,84 & 14,95 & 13,90 & 4,76 \\
\hline Fósforo $(\mathrm{P})$ & - & - & - & 2,3 & - & - & - & - & - \\
\hline Óxido de magnesio $(\mathrm{MgO})$ & $3-16$ & 5,4 & 11,7 & 1,45 & 4,02 & 9,08 & 8,58 & - & 3,65 \\
\hline Óxido de sodio $\left(\mathrm{Na}_{2} \mathrm{O}\right)$ & - & - & - & 1,4 & 1,06 & 0,56 & - & 0,65 & 2,55 \\
\hline Azufre $(\mathrm{S})$ & $1-3$ & 0,4 & - & 1,0 & - & - & - & - & - \\
\hline Óxido de hierro $(\mathrm{FeO})$ & $0.3-0.2$ & 0,2 & - & - & - & 0,6 & - & - & - \\
\hline Hierro $(\mathrm{Fe})$ & - & - & - & 0,5 & - & - & - & - & - \\
\hline
\end{tabular}




\begin{tabular}{|l|c|c|c|c|c|c|c|c|c|}
\hline Componente & {$[33]$} & {$[34]$} & {$[26]$} & {$[35]$} & {$[29]$} & {$[36]$} & {$[32]$} & {$[37]$} & {$[38]$} \\
\hline Óxido de hierro III $\left(\mathrm{Fe}_{2} \mathrm{O}_{3}\right)$ & - & - & 1,05 & 0,71 & 11 & - & 1,07 & 6,52 & 0,15 \\
\hline $\begin{array}{l}\text { Óxido de manganeso } \\
(\mathrm{MnO})\end{array}$ & $0,2-1,5$ & 0,5 & - & - & 2,62 & 0,5 & - & 2,26 & - \\
\hline Óxido de titanio $\left(\mathrm{TiO}_{2}\right)$ & - & 0,3 & - & - & 1,26 & - & - & 0,98 & - \\
\hline Óxido de potasio $\left(\mathrm{K}_{2} \mathrm{O}\right)$ & - & 0,4 & - & 0,14 & 0,51 & - & - & 0,86 & 0,62 \\
\hline
\end{tabular}

Fuente: elaboración propia

Tal como se observa en la tabla 1 , las BFS se componen principalmente de $\mathrm{SiO}_{2}$, $\mathrm{CaO}$ y $\mathrm{Al}_{2} \mathrm{O}_{3}$. Mineralógicamente, las $\mathrm{BFS}$ se componen principalmente de cuarzo $\left(\mathrm{SiO}_{2}\right)$ y la cristobalita (fase cúbica de alta temperatura del sílice $-\mathrm{SiO}_{2}$ ) [37]. $\mathrm{El} \mathrm{SiO}_{2}$ y el $\mathrm{Al}_{2} \mathrm{O}_{3}$ son compuestos puzolánicos los cuales, en tamaños de partículas muy finas, pueden desarrollar propiedades autocementantes (no necesitan ser activadas con agua o cal) [39]. Esta propiedad puzolánica puede incrementar la resistencia al daño por humedad de mezclas asfálticas [40]. Además, la presencia de un alto contenido de cuarzo en el BFS revela que este puede desarrollar una buena resistencia mecánica. Asimismo, el $\mathrm{SiO}_{2}$ y el $\mathrm{Al}_{2} \mathrm{O}_{3}$ pueden mejorar la adhesión superficial asfalto-agregado [41]. De acuerdo con [41, 42], el CaO es una composición alcalina que contribuye a aumentar la adhesión entre el asfalto y el agregado, y mejora la resistencia de las mezclas al daño por humedad y al stripping. [42] reporta que el $\mathrm{SiO}_{2}$ es un material de alta dureza y resistencia mecánica, y el $\mathrm{Al}_{2} \mathrm{O}_{3}$ puede mejorar la adherencia entre el agregado y el asfalto, incrementando la resistencia de las mezclas al ahuellamiento.

Con respecto a la utilización de BFS en la producción de mezclas asfálticas, muy pocos estudios han sido realizados en comparación con los otros usos reportados con anterioridad y con el empleo de SS. [24, 25, 37, 43] reportan que, al triturarse estos materiales, producen un agregado con una textura superficial rugosa de alta porosidad que resulta en buenas características adhesivas con ligantes asfálticos. Sin embargo, estas propiedades llevan a la necesidad de mayores cantidades de asfalto para producir una mezcla, generando un incremento en el costo [21, 25]. Adicionalmente, las BFS podrían precisar de mayores periodos de calentamiento en plantas de asfalto para secarlas [44]. A pesar de lo anterior, este mayor costo se podría compensar con el mayor rendimiento (volumen por masa) de BFS como agregado debido al menor peso unitario de la mezcla, ya que las BFS tienden a presentar menor densidad y gravedad específica en comparación con MGN [24, 37, 43]. En [37], utilizando un microscopio electrónico de barrido (MEB), se reportan que la porosidad promedio de una BFS osciló entre $9 \%$ y $63 \%$ mientras que un MGN fue del $7 \%$. Asimismo, el diámetro de las gargantas porales de la BFS fue mayor (entre 30,3 y 82,6 $\mu \mathrm{m}$ ) comparado con las de la MGN $(12 \mu \mathrm{m})$. 
Algunas conclusiones obtenidas por la FHWA [21] son: i) debido a su forma angular y textura superficial rugosa, las BFS generan un alto ángulo de fricción (entre $40^{\circ}$ y $45^{\circ}$, evaluadas a través de ensayos triaxiales [24]). Lo anterior ayuda a que, al ser usadas para conformar mezclas asfálticas en capas de rodadura, generen buena resistencia a la fricción neumático-pavimento. ii) Mezclas asfálticas fabricadas con BFS han demostrado buena resistencia al fenómeno del stripping y alta estabilidad. Incluso en algunos estudios se ha evidenciado buena resistencia a las deformaciones permanentes bajo carga cíclica [28]. Sin embargo, la resistencia al impacto y a la abrasión en algunos casos no es alta y el material puede descomponerse en condiciones de tráfico pesado [37]. Por lo anterior, estos materiales como agregados han sido más utilizados en la conformación de capas asfálticas de pavimentos con bajo tráfico. iii) La variabilidad en el proceso de producción de hierro puede dar lugar a heterogeneidad en las propiedades físicas (gradación, gravedad específica, absorción y angulosidad) y químicas (ver tabla 1). iv) Aunque la resistencia al desgaste por abrasión de las escorias de alto horno en la máquina de los ángeles no es particularmente favorable, el rendimiento de campo ha sido satisfactorio. Por tal razón, este ensayo ha sido eliminado de algunas especificaciones estándar para caracterizar escorias de alto horno. v) Tienen buena afinidad con el asfalto, lo que ayuda a desarrollar una buena adherencia. vi) Por ser un material poroso en comparación con agregados convencionales, puede ser necesario un tiempo de secado más largo en plantas de producción de mezclas asfálticas.

Con base en los resultados de desgaste en Micro-Deval y $10 \%$ de finos, en [37] reportan que las BFS presentan una buena resistencia al desgaste por abrasión por fricción entre partículas y al fracturamiento bajo carga monotónica, respectivamente. Sin embargo, tal como se reporta en estudios anteriores, las BFS son materiales que experimentan baja resistencia al desgaste por abrasión por impacto en la máquina de los ángeles. Asimismo, reportan, que dicho material presenta partículas con formas ideales (redondeadas con caras angulares y fracturadas con muy poco contenido de partículas alargadas y aplanadas) para desarrollar un buen esqueleto granular que ayude en procesos de compactación de mezclas asfálticas, así como a resistir cargas estáticas, dinámicas o de impacto. Las partículas finas no presentan contenido de arcilla, materia orgánica o de polvo que genere disminución de adherencia entre el agregado pétreo y el asfalto, y disminución de resistencia y durabilidad de la mezcla por exceso de finos o contaminantes.

En comparación con las SS, las BFS son volumétricamente más estables [25, 45]. Sin embargo, a largo plazo, estos materiales pueden tener problemas asociados a reacciones químicas con el agua (principalmente hidratación de partículas con propiedades puzolánicas presentes en las BFS que no han sido previamente activadas), ciclos de 
congelamiento-secado que pueden producir transformaciones químicas, entre otros factores, que generen agrietamientos y alteraciones en las partículas [46].

\section{ESCORIAS DE ACERO}

\subsection{Generalidades}

Las escorias de acero se producen en hornos básicos de oxigeno (BOF) y en hornos de arco eléctrico (EAF). De acuerdo con $[2,47]$ se producen en una cantidad entre el $10 \%-15 \%$ y $15 \%-20 \%$ en peso de la producción de acero, respectivamente. La BOF se produce soplando oxígeno al hierro fundido, el cual es mezclado con flujos adicionales y chatarra de acero reciclado. El proceso refina el hierro mediante fusión con un fundente, tal como piedra caliza o dolomita, en condiciones oxidantes. Las impurezas del hierro, tales como el carbono y el silicio, se oxidan o se combinan químicamente con la escoria. La EAF se genera durante la producción de aceros más especializados [25] y por lo general presenta mayor cantidad de hierro y menor contenido de óxidos de magnesio y calcio libres que la escoria BOF [3, 48, 49]. Lo anterior las hace ser más densas y resistentes a la abrasión, a cargas monotónicas y por impacto, que las BOF. Adicionalmente, a diferencia del proceso BOF, en las EAF no se utiliza metal caliente [30].

La cantidad de SS de diferentes industrias siderúrgicas es de 150 a $200 \mathrm{~kg} /$ tonelada de acero producido [26]. La producción anual de SS en China es de cerca de 17 millones de toneladas [50]. Según [51], en China cerca de 100 millones de toneladas de SS se generan todos los años y la cantidad de almacenamiento acumulado ha superado los 1,2 billones de toneladas. La producción anual de acero y SS en 2010 en China fue de 626,7 millones de toneladas y 90 millones de toneladas, respectivamente, y la tasa de utilización de SS fue del $22 \%$ [52]. Cincuenta millones de toneladas por año de SS se producen como subproducto en el mundo [53]. En Grecia, las tasas de producción anual de SS se han estimado en 1,6 millones de toneladas [49]. En Irán dicha tasa anual es de 3 millones [54]. En Letonia se producen anualmente entre 100 y $200 \mathrm{mil}$ toneladas de SS [55]. En Brasil, alrededor de 3 millones de toneladas por año de SS son generadas por las fábricas de acero [56]. La industria de fabricación de acero en los Estados Unidos genera de 9 a16 millones de toneladas de SS al año, de las cuales se utiliza como agregado pétreo en carreteras y en la construcción de pavimentos aproximadamente entre $50 \%$ y $70 \%$, y en otras aplicaciones diversas aproximadamente $10 \%$ a $15 \%$ [57, 58]. En Alemania más del $90 \%$ de las escorias son reutilizadas en proyectos viales [59]. En Europa se producen más de 12 millones de escorias al año [3]. En [11] reportan en Croacia, la existencia de vertederos con aproximadamente 1,5 millones de toneladas de SS. 
Algunos usos de las SS identificados son: producción de concreto [5, 60, 61], cemento y la construcción de carreteras [49], en estabilización de suelos y empleo como materiales granulares no tratados en diferentes aplicaciones y en pavimentos (base, subbase, afirmados, entre otros) [62-64], como fertilizantes [3, 52], como material adsorbente en el tratamiento de aguas residuales urbanas y aguas residuales industriales [65], en la agricultura [52, 66, 67], en la estabilización de bancas para los ríos [3], y en la recuperación de acero residual (se estima en un $10 \%$ [52]) y de metales [26]. En esta última aplicación, los contenidos de $\mathrm{Mn}, \mathrm{CaO}$ y $\mathrm{MgO}$ presentes en el hierro recuperado o reciclado de las SS podrían mejorar el nuevo hierro o acero obtenido a partir de dicho reciclaje [68].

\subsection{Propiedades de las SS y uso en mezclas asfálticas}

En la tabla 2 se presenta la composición química típica de las SS.

Tabla 2. Composición química de SS

\begin{tabular}{|c|c|c|c|c|c|c|c|c|c|c|c|}
\hline Componente & $\begin{array}{l}{[69]} \\
E A F\end{array}$ & $\begin{array}{l}{[70]} \\
E A F\end{array}$ & $\begin{array}{l}{[58]} \\
B O F\end{array}$ & {$[71]$} & $\begin{array}{l}{[52]} \\
E A F\end{array}$ & $\begin{array}{l}{[52]} \\
B O F\end{array}$ & $\begin{array}{l}{[72]} \\
E A F\end{array}$ & $\begin{array}{l}{[57]} \\
E A F\end{array}$ & $\begin{array}{l}{[30]} \\
E A F\end{array}$ & $\begin{array}{l}{[30]} \\
B O F\end{array}$ & $\begin{array}{l}{[60]} \\
E A F\end{array}$ \\
\hline $\mathrm{CaO}[\%]$ & $0,4-52$ & 25,58 & 39,4 & 50,5 & $30-50$ & $45-60$ & 33,2 & 37,78 & $35-60$ & $30-55$ & 27 \\
\hline $\mathrm{SiO}_{2}[\%]$ & $0,8-19$ & 18,72 & 11,97 & 14,4 & $11-20$ & $10-15$ & 10,08 & 16,47 & $9-20$ & $8-20$ & 11 \\
\hline $\mathrm{Al}_{2} \mathrm{O}_{3}[\%]$ & $1-14,07$ & 2,75 & 2,16 & 1,35 & $10-18$ & $1-5$ & 1,66 & 5,76 & $2-9$ & $1-6$ & 5 \\
\hline $\mathrm{P}[\%]$ & - & - & - & - & - & - & - & - & $0,01-0,25$ & $0.2-2$ & - \\
\hline $\mathrm{P}_{2} \mathrm{O}_{5}[\%]$ & $0,48-1,24$ & - & 1 & 1,1 & $2-5$ & $1-4$ & - & 0,76 & - & - & - \\
\hline $\mathrm{MgO}[\%]$ & $0,4-10,2$ & 7,50 & 1,69 & 4,5 & $8-13$ & $3-13$ & 13,09 & 4,81 & $5-15$ & $5-15$ & 5 \\
\hline Cromo $(\mathrm{Cr})[\%]$ & - & - & - & - & - & - & - & - & $0,1-1$ & $0,1-0,5$ & - \\
\hline $\mathrm{Na}_{2} \mathrm{O}[\%]$ & - & 0,29 & 0,25 & - & - & - & 0,02 & 0,06 & - & - & - \\
\hline $\mathrm{FeO}[\%]$ & $20,4-37,2$ & - & 30,23 & 14,8 & $8-22$ & $7-20$ & - & - & $15-30$ & $10-35$ & 25 \\
\hline $\mathrm{Fe}[\%]$ & $15-30$ & - & - & - & - & - & - & 0,02 & - & - & - \\
\hline $\mathrm{S}[\%]$ & - & - & - & - & - & - & - & - & $0,08-2$ & $\begin{array}{c}0,05- \\
0,15\end{array}$ & - \\
\hline $\mathrm{SO}_{3}[\%]$ & - & - & 0,12 & - & - & - & - & 0,34 & - & - & - \\
\hline $\mathrm{Fe}_{2} \mathrm{O}_{3}[\%]$ & $24,2-37,2$ & 35,16 & - & 5,2 & $5-6$ & $3-9$ & 29,64 & 31,02 & - & - & 25 \\
\hline $\mathrm{MnO}[\%]$ & $1,07-15,58$ & 0,304 & 2,74 & - & $5-10$ & $2-6$ & 6,18 & - & $3-8$ & $2-8$ & 4 \\
\hline $\mathrm{Cr}_{2} \mathrm{O}_{3}$ & $0,78-4,07$ & - & - & - & - & - & - & - & - & - & - \\
\hline $\mathrm{TiO}_{2}[\%]$ & $0,35-0,6$ & 1,595 & 0,4 & 0,5 & - & - & - & - & - & $0,4-2$ & - \\
\hline $\mathrm{K}_{2} \mathrm{O}[\%]$ & - & 0,13 & 0,05 & - & - & - & 0,06 & 0,02 & - & - & - \\
\hline
\end{tabular}

$\mathrm{P}_{2} \mathrm{O}_{5}$ : óxido de fosforo (III), $\mathrm{Cr}_{2} \mathrm{O}_{3}$ : óxido de cromo (III), $\mathrm{SO}_{3}$ : óxido de azufre (VI). 
En [59], basados en múltiples estudios consultados, se hacen reportes sobre nueve escorias BOF y once EAF, composición química similar a las reportadas en la tabla 2. Los resultados presentados en la tabla 2 son coherentes con los reportados por [30, 73] sobre cuatro tipos de SS estudiadas en Suecia y por [8, 50, 53, 74, 75]. Como se observa en la tabla 2, los principales componentes de las $\mathrm{SS}$ son $\mathrm{CaO}, \mathrm{Fe}, \mathrm{SiO}_{2}, \mathrm{MgO}$ y $\mathrm{MnO}$. Estos componentes le podrían atribuir a las SS alta dureza, resistencia a la abrasión, a cargas de impacto, monotónicas y cíclicas. Adicionalmente, la alta magnitud de la relación $\mathrm{CaO} / \mathrm{SiO}_{2}$ de las $\mathrm{SS}$ genera una mejor adherencia entre el agregado y el asfalto $[44,56,70,76,77]$. La composición química y la microtextura rugosa de las SS permite que se formen fuertes entrecruzamientos de partículas [78, 79] y enlaces adherentes con el asfalto [49, 50, 80-82]. En [59], basados en las propiedades hidrófobas de una BOF y un $\mathrm{pH}$ cercano a 12, señalan que este material proporciona buena adhesión con el asfalto y ayudan a resistir fenómenos como el stripping. En [54], basados en imágenes obtenidas con microscopía electrónica de barrido (SEM) de una escoria EAF, reportan que estos materiales pueden desarrollar una adhesión más fuerte con el asfalto en comparación con mezclas convencionales, principalmente por su porosidad. Una mayor adherencia de una escoria BOF con el asfalto, en comparación con basalto y granito, es también reportada por [83] basados en la composición mineralógica, química y en ensayos de tracción indirecta (empleando una máquina universal y analizando, como variables, dos espesores de película bituminosa, tres temperaturas de ensayo y tres tratamientos de humedad).

Según [84], no todas las SS son apropiadas para ser usadas como agregados en mezclas asfálticas. En especial aquellas ricas en $\mathrm{CaO}$ y $\mathrm{MgO}$, tienden a expandirse en ambientes húmedos por hidratación de dichos componentes químicos cuando estos no han reaccionado con las estructuras de silicato $[14,24,45,53,57,58,70,75$, 85-87]. Esta propiedad ha sido identificada por diversos investigadores y establecida como la principal limitación de uso de SS en la construcción de carreteras $[3,10$, $54,88]$. Sin embargo, algunos investigadores han reportado que este problema de expansión se puede evitar sometiendo las SS a un largo período de intemperismo o envejecimiento natural $[24,56,89]$ durante al menos un año antes de que se utilice como agregado secundario. Sin embargo, esta técnica puede ser penalizada por exponer al aire libre las SS al medio ambiente [90]. Con base en información proporcionada por un fabricante de escoria llamado Multiserv, [58], a manera de ejemplo, mencionan que el contenido típico de cal libre para una escoria BOF en estado inicial y envejecido durante mínimo un año de edad es de $14,5 \%$ y 4,5 \%, respectivamente. Es decir, sí existe una disminución en el contenido de cal libre cuando se envejecen este tipo de escorias. A pesar de lo anterior, en el mismo estudio concluyen que, en algunos casos, el envejecimiento de las escorias no necesariamente asegura su estabilidad volumétrica. Otra forma de evitar la expansión es recubriendo adecuadamen- 
te las partículas con una suficiente película de asfalto que no permita que el agua entre en contacto con el agregado [25]. Este recubrimiento también es de alta importancia porque en presencia de agua, los oxidos $\left(\mathrm{SiO}_{2}, \mathrm{Fe}_{2} \mathrm{O}_{3}, \mathrm{MgO}\right.$ y $\left.\mathrm{Al}_{2} \mathrm{O}_{3}\right)$ que componen las SS pueden reaccionar y separarse del agregado pétreo generando strippping [70]. En [91] mencionan que mezclar SS con Fayalita ayuda a inhibir la reacción de expansión. En [58] reportan que sustituir un $10 \%$ de una escoria BOF por ceniza volante del tipo C puede suprimir su comportamiento expansivo.

Al igual que las BFS, las SS presentan propiedades cementantes [29, 51, 92] debido a la presencia de C3S, C2S y C4AF [93]. En la tabla 3 se presentan algunas propiedades físicas de las SS.

Tabla 3. Propiedades físicas de SS.

\begin{tabular}{|l|c|c|c|c|c|c|c|c|c|}
\hline \multirow{2}{*}{ Ensayo } & \multirow{2}{*}{ Unidad } & \multicolumn{7}{|c|}{ Magnitud } \\
\cline { 4 - 11 } & & {$[94]$} & {$[71]$} & {$[95]$} & {$[70]$} & {$[56]$} & {$[96]$} & {$[97]$} & {$[60]$} \\
\hline Densidad & $\mathrm{g} / \mathrm{cm}^{3}$ & - & - & 3,3 & - & 3.92 & - & - & - \\
\hline Gravedad específica gruesos & - & - & 3,02 & - & 3,01 & - & - & 3,25 & 3,35 \\
\hline Gravedad específica finos & - & - & 3,17 & - & 3,06 & - & - & 3,15 & 3,7 \\
\hline Absorción & $\%$ & - & - & 1,29 & - & - & $2,9-3,1$ & $2,5-3,7$ & $2,84-3,29$ \\
\hline Caras fracturadas (dos caras) & $\%$ & - & - & - & 97,6 & - & - & 100 & - \\
\hline Partículas alargadas y aplanadas & $\%$ & - & 6,1 & - & - & - & $2-3$ & 1 & - \\
\hline Equivalente de arena & $\%$ & - & - & - & - & 83 & - & 86 & - \\
\hline $\begin{array}{l}\text { Desgaste en la máquina de los } \\
\text { ángeles }\end{array}$ & $\%$ & $20-25$ & 20 & 13,2 & 14,2 & $14-15$ & 24 & 18,6 & 20 \\
\hline Valor de agregado fracturado & $\%$ & - & - & - & - & - & 23 & - & - \\
\hline Resistencia a sulfato de sodio & $\%$ & $<12$ & - & - & - & - & 2,07 & - & - \\
\hline Ángulo de fricción interna & - & $40^{\circ}-50^{\circ}$ & - & - & - & - & - & - & - \\
\hline Dureza (escala de Moh's) & - & $6-7$ & - & - & - & - & - & - & - \\
\hline CBR & $\%$ & $>300$ & - & - & - & - & - & - & - \\
\hline Stripping & $\%$ & - & - & - & - & - & $>95 \%$ & - & - \\
\hline
\end{tabular}

Fuente: elaboración propia

En [59], basados en seis estudios consultados, se reportan magnitudes de gravedad específica, CBR y desgaste en la máquina de los ángeles similares a las reportadas en la tabla 3. Con base en la tabla 3 se concluye que la densidad de las SS es mayor que la de MGN. Lo anterior es debido principalmente a que las SS contienen alto contenido de hierro $[52,58,59,73]$. Esta propiedad podría aumentar el costo de transporte de la 
SS como agregado y de las mezclas fabricadas con dichos materiales. Adicionalmente, son materiales de alta dureza, densidad, absorción y resistencia a la abrasión $[5,49,50$, $53,55,58,69,81,85,88,94,98-102]$, presentan superficie rugosa y buena forma de partículas en cuanto a angularidad y caras fracturadas [54, 74]. La mayor resistencia a la abrasión es fundamentada por el alto contenido de $\mathrm{Fe}_{2} \mathrm{O}_{3}$ y $\mathrm{SiO}_{2}$ [70]. Debido a sus altos niveles de dureza y angularidad, mezclas fabricadas con SS han desarrollado mayor resistencia al deslizamiento (neumático-pavimento) en pruebas realizadas en laboratorio y a escala real sobre vías pavimentadas [1, 25, 47, 48, 80, 81, 85, 103]. Con base en ensayos realizados sobre tres agregados naturales producidos por diferentes canteras ubicadas en Brasil y un tipo de SS, en [104] se reporta que la SS es más resistente al desgaste por pulimento. De acuerdo con [97], la mayor dureza de las SS puede ser beneficial en países donde se utilizan neumáticos para reducir los accidentes relacionados con la nieve y el hielo en época de invierno. Según [73], las SS son difíciles de triturar y moler. En [52] reportan que el índice de molienda de las escorias de acero es menor $(0,7)$ comparado con las escorias de alto horno $(0,96)$ y arenas estándares $(1,0)$. Lo anterior incide en el costo de uso de las SS.

En [105] se menciona que, a pesar de que las partículas de SS en forma de nido de abeja son poco frecuentes, cuando estas están presentes en una mezcla asfáltica generan aumento en el contenido de asfalto y disminución en la resistencia a la deformación permanente, al daño por humedad y al agrietamiento.

Las SS retienen el calor mucho más que los MGN [80, 85, 87, 106, 107]. Las características de retención de calor de las SS pueden ser ventajosas para trabajos de reparación de mezclas asfálticas en caliente durante condición de clima frío [53, 108]. Adicionalmente, desarrollan mayor conductividad eléctrica. En [53] realizaron ensayos sobre una mezcla de concreto asfáltico donde se sustituyó la fracción gruesa de un material calcáreo por SS (se emplearon como ligantes cemento asfáltico tipo $\mathrm{AC}-5$ y AC-10). Los autores concluyen que las mezclas fabricadas con SS permiten su uso en la construcción de pavimentos termoeléctricos (pavimentos con materiales eléctricamente conductivos usados para aumentar el calor del mismo cuando se requiera) para el deshielo de garajes, aceras, calzadas, puentes de carreteras y pistas de aterrizaje.

$\mathrm{Al}$ igual que las $\mathrm{BFS}$, las SS presentan generalmente mayor porosidad y rugosidad superficial que MGN [76]. Debido a esto, al igual que las mezclas fabricadas con BFS, aquellas fabricadas con SS consumen mayor cantidad de asfalto, pudiendo incrementar el costo de las mismas $[80,101]$ y retienen más el agua durante los procesos de calentamiento y secado en las plantas de asfalto [44]. Sin embargo, algunos autores han reportado una disminución en la cantidad de asfalto como producto de la mayor gravedad específica de la escoria [109], y de que, en algunos casos y en algunos lugares, 
los MGN presentan mayor angularidad y absorción que las escorias de acero. En un estudio realizado por [94, 97], por ejemplo, la absorción de la fracción gruesa de la SS y el MGN de origen basáltico era de 2,5 \% y 2,0 \%, mientras que la fracción fina de 3.7 y $5.2 \%$, respectivamente.

Generalmente, el uso de SS en mezclas asfálticas debe limitarse a la sustitución parcial del agregado natural debido a que las mezclas con sustitución total son susceptibles a experimentar problemas asociados con el aumento del volumen de vacíos (Va) y disminución de los vacíos en agregados minerales (VAM). Lo anterior debido principalmente a su forma angular, porosidad y mayor textura superficial en comparación con MGN [10, 53, 101]. En [98] realizaron ensayos sobre una mezcla asfáltica en la cual sustituyeron el $100 \%$ de un agregado calcáreo por SS. Los resultados concluyen que usar $100 \%$ de sustitución no trae beneficios en algunas propiedades como la deformación permanente en clima de alta temperatura y resistencia a fatiga. En [100] reportan, que al sustituir completamente el MGN por una SS tipo BOF, los vacíos entre los agregados pétreos (VAM) disminuyen notablemente, generando un muy delgado recubrimiento de las partículas. En [10] hallaron una mayor resistencia a la deformación de mezclas fabricadas con SS en comparación con la mezcla de control, excepto para la sustitución del agregado grueso de origen calcáreo al $100 \%$ (ellos plantean que con el $100 \%$ de sustitución se necesita mayor cantidad de asfalto). Adicionalmente, mencionan que el porcentaje óptimo de reemplazo fue de $25 \%$.

Generalmente, el uso de SS como agregado en mezclas asfálticas, incrementa la estabilidad Marshall, la relación estabilidad/flujo y disminuye el flujo [1, 53, 54, 76, 101, $110]$. En $[8,74]$, reportan que mezclas fabricadas con SS desarrollan mayor resistencia en el ensayo de tracción indirecta que mezclas fabricadas con agregados convencionales. En $[53,56,89,97]$ encontraron que la adición de SS en el concreto asfáltico mejora la resistencia a la deformación permanente de las mezclas. Una conclusión similar fue reportada por [76] para el caso de una mezcla tibia (WMA). De acuerdo con [1, 101], el módulo resiliente de mezclas asfálticas incrementa cuando se sustituyen MGN por SS. En [1] realizaron un estudio sustituyendo un agregado calcáreo por dos SS tipo EAF en $0 \%, 30 \%, 60 \%$ y $90 \%$ (con respecto a la masa total del agregado). La sustitución incluía partículas gruesas y finas del agregado. Se encontró que todas las mezclas de escoria exhibían una mejor resistencia a la deformación permanente que la mezcla de control bajo cargas axiales repetidas. En [1,56], basados en ensayos RLAT (Repeated Load Axial Test) concluyen que las mezclas con SS tienen poca probabilidad de desarrollar deformaciones permanentes excesivas. En [88], basados en ensayos realizados sobre mezclas asfálticas recicladas en frío, concluyeron que al reemplazar entre el $10 \%$ y el $20 \%$ del peso de un MGN, la resistencia a fatiga evaluada en un ensayo de tensión 
indirecta incrementa. Una conclusión similar fue reportada por [53] cuando se sustituyó, en una mezcla de concreto asfáltico, el $100 \%$ de la fracción gruesa de un MGN calcáreo por SS, y por [10] cuando sustituyeron también dicha fracción de un material calcáreo en $25 \%, 50 \%, 75 \%$ y $100 \%$. En [98] concluyeron que mezclas fabricadas con un asfalto modificado con SBS (estireno-butadieno-estireno) y empleando SS como sustituto de la fracción gruesa de un material calcáreo, desarrollan una vida de fatiga elevada y una alta resistencia a la deformación permanente. En [54], basados en ensayos de fatiga realizados mediante tres metodologías distintas, confirman que las mezclas que contienen escoria tipo EAF, tienen una vida a fatiga mayor que las mezclas de control que emplean MGN calcáreo. Según los autores, dicho aumento en la resistencia a fatiga es causado por la alta angularidad de la escoria (aumentando el ángulo de fricción interno de la mezcla y mejorando el entrelazamiento entre las diferentes partículas constituyentes), el mejoramiento en la adherencia entre el asfalto y la SS, y el incremento en la resistencia al envejecimiento que se desarrolla. Lo anterior también pudo ser debido a un mejor recubrimiento de los agregados producto de un mayor contenido de asfalto y VMA reportado en el diseño de las mezclas cuando se sustituyó el agregado natural por la EAF. Otros investigadores que han reportado un incremento en la resistencia a fatiga cuando se sustituye MGN por SS son [56].

En un estudio realizado por [47], se emplearon técnicas de difracción de rayos X (DRX), SEM y porosimetría de intrusión de mercurio (MIP) para evaluar el uso de SS como agregado en una mezcla tipo SMA (Stone Mastic Asphalt). Los resultados indicaron que la sustitución de basalto por SS aumentó la resistencia de la SMA a altas temperaturas de servicio (mayor resistencia a las deformaciones permanentes) y al agrietamiento a bajas temperaturas.

De acuerdo con $[1,10,53,70,76,77,88]$, la resistencia al daño por humedad incrementa en mezclas asfálticas cuando se emplean SS como parte del agregado pétreo. En [50], realizaron un estudio para evaluar el efecto de una SS tipo BOF cuando sustituye agregados gruesos de origen basáltico y calcáreo sobre la susceptibilidad al daño por humedad de una mezcla tipo SMA. Los autores reportaron que la SS no tiene un efecto significativo sobre el daño por humedad.

En $[94,97]$ utilizaron cuatro porcentajes $(0 \%, 20 \%, 40 \%$ y $60 \%)$ de SS para sustituir un agregado basáltico, y los diseños de mezcla para HMA se realizaron con base en la metodología Superpave. Los resultados indican que la adición de SS incrementa la fricción neumático-pavimento, el módulo dinámico, la resistencia al agrietamiento por bajas temperaturas y la resistencia a la deformación permanente. Además, la inclusión de SS tuvo poco efecto sobre la resistencia al agrietamiento TDC (Top-Down-Cracking) y sobre la susceptibilidad al daño por humedad. 
En [107] compararon los resultados de analizar el comportamiento de una vía construida con capa asfáltica con MGN y otra construida con una escoria BOF. Se realizaron ensayos Marshall, resistencia a la tracción indirecta, módulo elástico y ensayos in situ para medir la resistencia al deslizamiento. El comportamiento de ambas mezclas, luego de medio año de servicio, fue igual. Sin embargo, después de dos años de servicio, el comportamiento de la capa asfáltica fabricada con SS fue mucho mejor que la convencional con MGN.

Li et al. [111] emplearon tres tipos de SS como filler para evaluar la respuesta reológica que experimentaban los mastic fabricados a partir dichos materiales. Como conclusión general del estudio, se reportó que los mastic con SS presentaron propiedades reológicas sobresalientes a altas y bajas temperaturas de servicio (mayor resistencia a las deformaciones permanentes y agrietamiento, respectivamente) cuando fueron comparados con un mastic fabricado a partir de un filler de origen calcáreo. Una conclusión similar fue reportada por Topini et al. [112] empleando una SS tipo EAF.

\section{CONCLUSIONES}

Con base en la revisión bibliográfica reportada y descrita en el presente estudio se puede concluir, desde el punto de vista técnico y ambiental, que: i) las escorias BFS y SS son materiales con un amplio potencial para ser empleados como sustitutos de agregados pétreos naturales en la fabricación de mezclas asfálticas. Incluso actualmente diversos países en el mundo las emplean para tal fin. En la mayoría de los estudios, ambas escorias cumplieron los requisitos mínimos de calidad exigidos por cada especificación particular empleada, y presentaron propiedades deseables en proyectos viales como son partículas predominantemente redondeadas con caras angulares, fracturadas y escaso contenido de partículas alargadas y aplanadas (indicadores de un esqueleto granular más compacto y menos deformable bajo carga mecánica), un equivalente de arena alto (indicador de un material que no tendrá exceso de finos), las partículas finas no presentaban contenido de arcilla o materia orgánica (estos dos materiales tienden a disminuir la respuesta mecánica de materiales granulares en proyectos viales), y en el caso de las SS alta resistencia al desgaste por abrasión. ii) En términos generales no se reportan riesgos ambientales o toxicológicos por usar BFS o SS. Sin embargo, en cada proyecto vial o uso particular se debe monitorear la concentración de algunos elementos que pueden ser nocivos para los seres humanos como son el vanadio, el cromo y el aluminio. iii) A pesar del amplio conocimiento que se tiene sobre sus propiedades y de las múltiples investigaciones realizadas, son materiales altamente heterogéneos en cuanto a formas y tamaños de partículas, composición química y mineralógica, textura, gravedad específica, resistencia al desgaste y porosidad, entre otros, los cuales deben seguirse estudiando. Por tal motivo, algunas propiedades evaluadas en los estudios consultados reportaron respuestas distintas y en algunos casos contradictorias. iv) El 
uso de BFS y SS podría ayudar a reducir el empleo de agregados pétreos naturales y disminuir el consumo de energía durante el proceso de extracción de dichos agregados. v) El principal componente químico que presentan ambas escorias es el $\mathrm{CaO}$, el cual puede aumentar la adhesión asfalto-agregado y mejorar la resistencia al daño por humedad. En algunas ocasiones ambas escorias presentan altos contenidos de $\mathrm{Al}_{2} \mathrm{O}_{3}$ el cual también es un óxido que contribuye a mejorar las propiedades mencionadas anteriormente y puede incrementar la resistencia de las mezclas al ahuellamiento. El contenido de $\mathrm{SiO}_{2}$ reportado en ambas escorias y de hierro en las SS puede ayudar a aumentar la cohesión y la resistencia mecánica de la mezcla (debido a que son materiales de alta dureza). Sin embargo, si el contenido de $\mathrm{SiO}_{2}$ es alto o se presenta una baja relación $\mathrm{CaO} / \mathrm{SiO}_{2}$, la adherencia agregado-asfalto podría verse reducida. vi) La principal limitación de uso de las BFS en proyecto viales es su baja resistencia a la abrasión, mientras que en el caso de las SS es su potencial de expansión. Asimismo, otra desventaja reportada es que, debido a su mayor porosidad y rugosidad superficial en comparación con agregados naturales, las mezclas que emplean BFS o SS tienden a necesitar mayor cantidad de asfalto. Esta es una de las principales razones por las cuales, en los estudios, generalmente, no se recomienda sustituir totalmente los agregados naturales por escorias BFS o SS. Investigaciones futuras se deben encaminar a la superación de estas limitaciones. vii) Las SS tienden a desarrollar propiedades termo-eléctricas (retienen el calor mucho más que los agregados naturales y desarrollan mayor conductividad eléctrica) y cementantes.

A continuación, se reportan algunas líneas de investigación en las cuales se debe profundizar aún más en el área de mezclas asfálticas:

- Muy pocos estudios han sido ejecutados para evaluar la relación costo-beneficio desde el punto de vista técnico y ambiental y que tengan en cuenta, adicionalmente, las condiciones propias de cada país donde se utilicen estos materiales.

- Por lo general, los estudios que se han realizado han sustituido el agregado pétreo natural por escorias en diversos porcentajes. Sin embargo, se debe evaluar con mayor detalle el efecto de los tamaños de partículas que se están sustituyendo. Asimismo, evaluar con mayor claridad el efecto de la granulometría, la forma, textura, porosidad y la gravedad específica de las partículas. Esta última propiedad es importante porque controla en gran medida la composición gravimétrica y volumétrica de la mezcla.

- Por lo general, los estudios consultados evalúan por separado el empleo de BSF y SS como materiales para fabricar mezclas asfálticas. Se deben realizar mayores investigaciones donde se analice el empleo de ambos materiales en distintas fracciones del agregado pétreo. 
- Gran parte del comportamiento que experimenta una mezcla asfáltica está asociado a la respuesta que desarrolla el mastic (filler y asfalto) que la compone. Sin embargo, muy pocos estudios se han ejecutado con el fin de evaluar la respuesta reológica y mecánica del mastic fabricado a partir de BSF o SS.

- Muy pocos estudios han evaluado la interacción asfalto-escoria. El asfalto, al entrar en contacto con la escoria, reacciona de manera diferente a como lo hace con agregados pétreos naturales y muy pocos estudios se han ejecutado en este sentido.

- Ningún estudio consultado se ejecutó para determinar las temperaturas de mezcla y compactación adecuadas en el laboratorio e in situ. Por ser las BFS y SS materiales con composición química y mineralógica diferentes a agregados naturales, y de alta porosidad y absorción, dichas temperaturas podrían ser diferentes a las utilizadas con MGN. Asimismo, se deben correlacionar estas temperaturas con el tipo de compactación (por ejemplo, impacto-Marshall, compactación giratoriaSuperpave) y con los tiempos de secado de BFS y SS en plantas de asfalto.

- Se deben explorar más metodologías y procedimientos para el control de la expansión de las escorias SS. Adicionalmente, se deben realizar más investigaciones para evaluar el impacto ambiental derivado de exponer las SS a prolongados periodos de intemperismo con el fin de disminuir su expansividad, que es el método más utilizado actualmente para controlar dicha limitación en las escorias.

- En comparación con las SS, muy pocos estudios han evaluado el efecto de la composición química de las BFS sobre las propiedades reológicas y mecánicas de mezclas asfálticas que emplean este material como agregado.

- Se deben hacer mayores estudios para evaluar a largo plazo las propiedades de estos materiales y cómo inciden en mezclas asfálticas.

\section{REFERENCIAS}

[1] M. Pasetto y N. Baldo, "Experimental evaluation of high performance base course and road base asphalt concrete with electric arc furnace steel slags," Journal of Hazardous Materials, vol. 181, N. ${ }^{\circ} 1-3$, pp. 938-948. 2010. DOI: 10.1016/j.jhazmat.2010.05.104.

[2] D.M. Proctor, K.A. Fehling, E.C. Shay, J.L. Wittenborn, J.J. Green, C. Avent, R.D. Bigham, M. Connolly, B. Lee, T.O. y Shepker, M.A. Zak, "Physical and chemical characteristics of blast furnace, basic oxygen furnace, and electric arc furnace steel industry slags," Environmental Science and Technology, vol. 34, N. ${ }^{\circ}$ 8, pp. 1576-1582. 2000. DOI: 10.1021/es9906002.

[3] H. Motz y J. Geiseler, "Products of steel slags an opportunity to save natural resources," Waste Management, vol. 21, pp. 285-293. 2001. DOI: 10.1016/S0956-053X(00)00102-1. 
[4] H. Shen y E. Forssberg, "An overview of recovery of metals from slags," Waste Management, vol. 23, pp. 933-949. 2003. DOI: 10.1016/S0956-053X(02)00164-2.

[5] M. Maslehuddin, A.M. Sharif, M. Shameem, M. Ibrahim y M.S. Barry, "Comparison of properties of steel slag and crushed limestone aggregate concretes," Construction and Building Materials, vol. 17, N. ${ }^{\circ}$ 2, pp. 105-112. 2003. DOI: 10.1016/S0950-0618(02)00095-8.

[6] S. Nouvion, A. Jullien, M. Sommier y V. Basuyau, "Environmental modeling of blast furnace slag aggregate production,” Road Materials and Pavement Design, vol. 10, N. ${ }^{\circ}$, pp. 715-745. 2009.

[7] J. Geiseler, "Use of steelworks slag in Europe," Waste Management, vol. 16, N. ${ }^{\circ} 1-3$, pp. 59-63. 1996. DOI: 10.1016/S0956-053X(96)00070-0.

[8] M. Pasetto y N. Baldo, "Mix design and performance analysis of asphalt concretes with electric arc furnace slag," Construction and Building Materials, vol. 25, N. ${ }^{\circ}$ 8, pp. 3458-3468. 2011. DOI: 10.1016/j.conbuildmat.2011.03.037.

[9] L. Wintenborn y J. Green, "Steelmaking slag: a safe and valuable product," National Slag Association. 1998.

[10] I.M. Asi, H.Y. Qasrawi y F.I. Shalabi, "Use of steel slag aggregate in asphalt concrete mixes," Canadian Journal of Civil Engineering, vol. 34, pp. 902-911. 2007. DOI: 10.1139/L07-025.

[11] I. Barišić, I.N. Grubeša y B.H. Kutuzović. "Multidisciplinary approach to the environmental impact of steel slag reused in road construction," Road Materials and Pavement Design, vol. 18, pp. 1-16. 2016. DOI: 10.1080/14680629.2016.1197143.

[12] A. Prapidis y G. Doulis, "Use of slag in skid resistant asphalt mixes based on mechanical and environmental criteria," Presentado en Proc. 5th International Exhibition \& Conference on Environmental Technology, Athens, Greece. 2005.

[13] A.M. Fällman, "Leaching of chromium and barium from steel slag in laboratory and field tests—a solubility controlled process?," Waste Management, vol. 20, $\quad$ N. ${ }^{\circ}$ 2, pp. 149-154. 2000. DOI: 10.1016/S0956-053X(99)00313-X.

[14] S. Sorlini, A. Sanzeni y L. Rondi, "Reuse of steel slag in bituminous paving mixtures," Journal of Hazardous Materials, vol. 209-210, pp. 84-91. 2012. DOI: 10.1016/j.jhazmat.2011.12.066.

[15] P. Chaurand, J. Rose, V. Briois, L. Olivi, J.-L. Hazemann, O. Proux, J. Domas y J.-Y. Bottero, "Environmental impacts of steel slag reused in road construction: a crystallographic and molecular (XANES) approach,” Journal of Hazardous Materials, Vol. 139, N. 3, pp. 537-542. 2007. DOI: 10.1016/j.jhazmat.2006.02.060.

[16] R. Milačič, T. Zuliani, T. Oblak, A. Mladenovič, A. and J.Š. Ančar, (2011). "Environmental impacts of asphalt mixes with electric arc furnace steel slag," Journal of Environmental Quality, vol. 40, N. ${ }^{\circ}$ 4, pp. 1153-1161. DOI: $10.2134 /$ jeq2010.0516.

[17] J. Yan, C. Bäverman, L. Moreno y I. Neretnieks, "Evaluation of the time-dependent neutralising behaviours of MSWI bottom ash and steel slag," Science of the Total Environment, vol. 216, N. ${ }^{\circ} 1-2$, pp. 41-54, 1998. DOI: 10.1016/S0048-9697(98)00133-8. 
[18] G. S. Roadcap, W. R. Kelly y C. M. Bethke, "Geochemistry of extremely alkaline (pH> 12) ground water in slag - fill aquifers," Ground Water, vol. 43, N. ${ }^{\circ} 6$, pp. 806-816, 2005. DOI: 10.1111/j.1745-6584.2005.00060.x.

[19] H. Seron Pereira, A.J. Manzi Gama, M. Sartori de Camargo y G.H. Korndorfer, "Reatividade de escórias silicatadas da indústria siderúrgica," Ciênc. Agrotec., lavras, vol. 34, N. ․ 2, pp. 382-390, 2010.

[20] D.M. Proctor, E.C. Shay, K.A. Fehling y B.L. Finley, "Assessment of human health and ecological risks posed by the uses of steel-industry slags in the environment," Human and Ecological Risk Assessment, vol. 8, N. ${ }^{\circ}$ 4, pp. 681-711, 2002.

[21] FHWA - Federal Highway Administration Research and Technology. Coordinating, Developing, and Delivering Highway Transportation Innovations, User Guidelines for Waste and Byproduct Materials in Pavement Construction, Report Publication Number: FHWA-RD-97-148, 2008.

[22] J.L. Marriaga y P. Claisse, "The influence of the blast furnace slag replacement on chloride penetration in concrete”. Ingeniería e Investigación, vol. 31, N. ${ }^{\circ}$ 2, pp. 38-47, 2011.

[23] NSA, National Slag Association, [En línea], acceso abril de 2016, Disponible: http://nationalslag.org/blast-furnace-slag

[24] H.G. Van Oss, Slag-Iron and Steel, U.S. Geologycal Survey Minerals Yearbook, Servicio Geológico de los Estados: USGS, 2003.

[25] G.D. Airey, A.C. Collop y N.H. Thom, "Mechanical performance of asphalt mixtures incorporating slag and glass secondary aggregates," Presentado en 8th Conference on Asphalt Pavements for Southern Africa (CAPSA'04), 2004.

[26] B. Das, S. Prakash, P.S.R. Reddy y V.N. Misra. "An overview of utilization of slag and sludge from steel industries," Resources, Conservation and Recycling, vol. 50, pp. 40-57, 2007. DOI: 10.1016/j.resconrec.2006.05.008.

[27] A. Jamshidi, K. Kurumisawa, T. Nawa, M. Jize y G. White, "Performance of pavements incorporating industrial byproducts: a state-of-the-art study," Journal of Cleaner Production, vol. 164, pp. 367-388, 2017. DOI: 10.1016/j.jclepro.2017.06.223.

[28] R. Dippenaar. "Industrial uses of slag (the use and re-use of iron and steelmaking slags)," Ironmaking and Steelmaking, vol. 32, N. ${ }^{\circ}$ 1, pp. 35-36, 2005.

[29] L.J.M. Houben, S. Akbarnejad y A.A.A. Molenaar, "Performance of pavements with blast furnace base courses," Presentado en GeoShanghai 2010 -International Conference, Paving Materials and Pavement Analysis, Geotechnical Special Publication N. 203 (pp. 476-483), China, 2010.

[30] C. Shi, "Steel slag - its production, processing, characteristics, and cementitious properties," Journal of Materials in Civil Engineering, vol. 16, N. 3, pp. 230-236, 2004. DOI: 10.1061/ (ASCE)0899-1561(2004)16:3(230).

[31] S.I. Abu-Eishah, A.S. El-Dieb y M.S. Bedir, "Performance of concrete mixtures made with electric arc furnace (EAF) steel slag aggregate produced in the Arabian Gulf 
region," Construction and Building Materials, vol. 34, pp.249-256, 2012. DOI: 10.1016/j. conbuildmat.2012.02.012.

[32] X. Guo y H. Shi, "Utilization of steel slag powder as a combined admixture with ground granulated blast-furnace slag in cement based materials," Journal of Materials in Civil Engineering. vol. 25, N. ${ }^{\circ}$ 12, pp. 1990-1993, 2013. DOI: 10.1061/(ASCE)MT.1943-5533.0000760.

[33] J.J. Emery, "Slag Utilization in Pavement Construction," Presentado en Extending aggregate resources: a symposium sponsored by ASTM Committee D-4 on Road and Paving Materials, EE. UU., 1982. DOI: 10.1520/STP32459S.

[34] D.E. Jones, “Application of steel plant by-products to roadworks," Presentado en Proceedings —Conference of the Australian Road Research Board, v 11, 11th ARRB Conference, 1982.

[35] N.A. León, N.R. Rojas, B.U. Suárez y O. Bustamante, "Experimental evaluation of silicon - calcareous units from blast furnace slag and hydraulic lime for masonry". Dyna, vol. 76, N. 160 , pp. 247-254, 2009.

[36] Q. Wang, P. Yan y G. Mi, "Effect of blended steel slag-GBFS mineral admixture on hydration and strength of cement," Construction and Building Materials, vol. 35, pp. 8-14, 2012. DOI: 10.1016/j.conbuildmat.2012.02.085.

[37] H.A. Rondón, J.C., Ruge, D., Patiño, H. Vacca, F.A. Reyes y M. Farias, "Use of blast furnace slag as a substitute for the fine fraction of aggregates in an asphalt mixture," Journal of Materials in Civil Engineering, vol. 30, N. 10, 04018244, 2018. doi:10.1061/(ASCE) MT.1943-5533.0002409

[38] A. Al-Hdabi y H. Al Nageim, "Improving asphalt emulsion mixtures properties containing cementitious filler by adding GGBS," Journal of Materials in Civil Engineering, vol. 29, ․․ 5, 04016297-1, 2016. doi:10.1061/(ASCE)MT.1943-5533.0001859.

[39] A. Misra, D. Biswas y S. Upadhyaya, "Physico-mechanical behavior of self-cementing class C fly ash-clay mixtures," Fuel, vol. 84, N. 11, pp. 1410-1422, 2005. DOI: 10.1016/j. fuel.2004.10.018.

[40] A.I. Nassar, M.K. Mohammedb, N. Thom y T. Parry, "Mechanical, durability and microstructure properties of Cold Asphalt Emulsion Mixtures with different types of filler," Construction and Building Materials, vol. 114, pp. 352-363, 2016. DOI: 10.1016/j.conbuildmat.2016.03.112.

[41] A. Modarres y M. Rahmanzadeh, "Application of coal waste powder as filler in hot mix asphalt," Construction and Building Materials, vol. 66, pp. 476-483. 2014. DOI: 10.1016/j. conbuildmat.2014.06.002.

[42] R. Muniandy, E. Aburkaba y L. Mahdi, "Effect of mineral filler type and particle size on asphalt-filler mastic and stone mastic asphalt laboratory measured properties," Australian Journal of Basic and Applied Sciences, vol. 7, N. ${ }^{\circ}$ 11, pp. 475-787, 2013.

[43] T. Ozbakkaloglu, L. Gu y A.F. Pour, "Normal and high-strength concretes incorporating air-cooled blast furnace slag coarse aggregates: Effect of slag size and content on the behavior," Construction and Building Materials, vol. 126, pp. 138-146, 2016. DOI: 10.1016/j. conbuildmat.2016.09.015. 
[44] J. Xie, S. Wu, J. Lin, J. Cai, Z. Chen y W. Wei, "Recycling of basic oxygen furnace slag in asphalt mixture: Material characterization and moisture damage investigation," Construction and Building Materials, vol. 36, pp. 467-474, 2012. DOI: 10.1016/j.conbuildmat.2012.06.023.

[45] G. Wang, Y. Wang y Z. Gao, "Use of steel slag as a granular material: Volume expansion prediction and usability criteria," Journal of Hazardous Materials, vol. 184, N. ${ }^{\circ} 1-3$, pp. 555-560, 2010. DOI: 10.1016/j.jhazmat.2010.08.071.

[46] S. Akbarnejad, L.J.M. Houben y A.A.A. Molenaar, "Application of aging methods to evaluate the long-term performance of road bases containing blast furnace slag materials," Road Materials and Pavement Design, vol. 15, N. ${ }^{\circ}$ 3, pp. 488-506, 2014. DOI: 10.1080/14680629.2014.907196.

[47] S. Wu, Y. Xue, Q. Ye y Y. Chen, "Utilization of steel slag as aggregates for stone mastic asphalt (SMA) mixtures," Building and Environment, vol. 42, ․․ 7, pp. 2580-2585, 2007. DOI: 10.1016/j.buildenv.2006.06.008.

[48] A.M. Dunster, "The use of blastfurnace slag and steel slag as aggregates," Presentado en 4th European symposium on performance of bituminous and hydraulic materials in pavements, Nottingham, 11-12 april of 2002. pp. 257-260.

[49] F. Kehagia, "Skid resistance performance of asphalt wearing courses with electric arc furnace slag aggregates," Waste Management and Research, vol. 27, N. ${ }^{\circ}$ 3, pp. 288-294, 2009. DOI: 10.1177/0734242X08092025.

[50] Y. Xue, S. Wu, H. Hou y J. Zha, "Experimental investigation of basic oxygen furnace slag used as aggregate in asphalt mixture," Journal of Hazardous Materials, vol. 138, N. ${ }^{\circ}$, pp. 261-268, 2006. DOI: 10.1016/j.jhazmat.2006.02.073.

[51] J. Zhao, D. Wang, P. Yan, D. Zhang y H. Wang, "Self-cementitious property of steel slag powder blended with gypsum," Construction and Building Materials, vol. 113, pp. 835-842, 2016. DOI: 10.1016/j.conbuildmat.2016.03.102.

[52] H. Yi, G. Xu, H. Cheng, J. Wang, Y. Wan y H. Chen, “An overview of utilization of steel slag,” Procedia Environmental Sciences, vol. 16, pp. 791-801, 2012. DOI: 10.1016/j. proenv.2012.10.108.

[53] P. Ahmedzade y B. Sengoz, "Evaluation of steel slag coarse aggregate in hot mix asphalt concrete," Journal of Hazardous Materials, vol. 165, pp. 300-305, 2009. DOI: 10.1016/j. jhazmat.2008.09.105.

[54] A. Kavussi y M.J. Qazizadeh, "Fatigue characterization of asphalt mixes containing electric arc furnace (EAF) steel slag subjected to long term aging," Construction and Building Materials, vol. 72, pp. 158-166, 2014. DOI: 10.1016/j.conbuildmat.2014.08.052.

[55] V. Haritonovs, M. Zaumanis, G. Brencis y J. Smirnovs, "Use of unconventional aggregates in hot mix asphalt concrete," Baltic Journal of Road and Bridge Engineering, vol. 9, N. ${ }^{\circ}$, pp. 276-282, 2014. DOI: 10.3846/bjrbe.2014.34.

[56] M. De Oliveira Polese, G. Lopes Carreiro, M. Gomes da Silva y M. Ribas Silva, "Caracterização Microestrutural da Escória de Aciaria,” Revista Matéria, vol. 11, N. ${ }^{\circ}$, pp. 444-454, 2006. 
[57] I.Z. Yildirim y M. Prezzi, "Geotechnical properties of fresh and aged basic oxygen furnace steel slag," Journal of Materials in Civil Engineering, vol. 27, N. ${ }^{\circ}$ 12, 2015. DOI: 10.1061/ (ASCE)MT.1943-5533.0001310.

[58] M. Pasetto y N. Baldo, "Laboratory investigation on foamed bitumen bound mixtures made with steel slag, foundry sand, bottom ash and reclaimed asphalt pavement," Road Materials and Pavement Design, vol. 13, N. ${ }^{\circ}$ 4, pp. 691-712, 2012. DOI: 10.1080/14680629.2012.742629.

[59] M.M.A. Aziz, M.R. Hainin, H. Yaacob, Z. Ali, F.L. Chang y A.M. Adnan, "Characterization and utilization of steel slag for the construction of roads and highways," Materials Research Innovations, vol. 18, S6, pp. 255-259, 2014. DOI: 10.1179/1432891714Z.000000000967.

[60] J.M. Manso, J.J. González y J.A. Polanco, “Electric arc furnace slag in concrete,” Journal of Materials in Civil Engineering, vol. 16, N. ${ }^{\circ}$ 6, pp. 639-645, 2004. DOI: 10.1061/ (ASCE)0899-1561(2004)16:6(639).

[61] H. Qasrawi, "The use of steel slag aggregate to enhance the mechanical properties of recycled aggregate concrete and retain the Environment," Construction and Building Materials, vol. 54, pp. 298-304, 2014. DOI: 10.1016/j.conbuildmat.2013.12.063.

[62] S. Pamukcu y A. Tuncan, "Laboratory characterization of cementstabilized iron-rich slag for reuse in transportation facilities," Transportation Research Record, N. ${ }^{\circ} 1424$, pp. 25-33, 1993.

[63] J.M. Manso, V. Ortega, J.A. Polanco y J. Setién, "The use of ladle furnace slag in soil stabilization," Construction and Building Materials, vol. 40, pp. 126-34. 2013. DOI: 10.1016/j. conbuildmat.2012.09.079.

[64] S. Aiban, "Utilization of steel slag aggregate for road bases," Journal of Testing and Evaluation, vol. 34, N. ${ }^{\circ}$ 1, pp. 1-11, 2006. DOI: 10.1520/JTE12683.

[65] S. Hosseini, S.M. Soltani, P.S. Fennell, T.S.Y. Choong y M.K. Aroua, "Production and applications of electric-arc-furnace slag as solid waste in environmental technologies: a review," Environmental Technology Reviews, vol. 5, N. 1, pp. 1-11, 2016. DOI: 10.1080/21622515.2016.1147615.

[66] R.M. Prado, W. Natale, F.M. Fernandes y M.C.M. Corrêa, "Reatividade de uma escória de siderurgia em um latossolo vermelho distrófico," R. Bras. Ci. Solo, vol. 28, pp. 197-205, 2004.

[67] M.F. Sobral, C.W.A. do Nascimento, K.P.V. da Cunha, H.A. Ferreira, A.J. Silva y F.B.V. Silva, "Escória de siderurgia e seus efeitos nos teores de nutrientes e metais pesados em cana-de-açúcar," Revista Brasileira de Engenharia Agrícola e Ambiental, vol. 15, N. 8 , pp.867-872, 2011.

[68] S. Radosavljevic, D. Milic and M. Gavrilovski, "Mineral processing of a converter slag and its use in iron ore sintering," Magnetic and Electrical Separation, vol. 7, N. ${ }^{\circ}$ 4, pp. 201-211, 1996. DOI: $10.1155 / 1996 / 31471$.

[69] M. Sumayya, M. Romeela y K. Prakash, "Characterization of electric arc furnace slags as concrete aggregate in a small island developing state: A preliminary study," Construction and Building Materials, vol. 105, pp. 459-464, 2016. DOI: 10.1016/j.conbuildmat.2015.12.169. 
[70] S. Hesami, M. Ameri, H. Goli y A. Akbari, "Laboratory investigation of moisture susceptibility of warm-mix asphalt mixtures containing steel slag aggregates," International Journal of Pavement Engineering, vol. 16, N. 8, pp. 745-759, 2015. DOI: 10.1080/10298436.2014.953502.

[71] M. Arabani y A.R. Azarhoosh, "The effect of recycled concrete aggregate and steel slag on the dynamic properties of asphalt mixtures," Construction and Building Materials, Vol. 35, pp. 1-7, 2012. DOI: 10.1016/j.conbuildmat.2012.02.036.

[72] T. Sofilić, A. Mladenovič y U. Sofilić, "Defining of EAF steel slag application possibilities in asphalt mixture production," Journal of Environmental Engineering and Landscape Management, vol. 19, N. ${ }^{\circ}$ 2, pp. 148-157, 2011. DOI: 10.3846/16486897.2011.580910.

[73] M. Tossavainen, F. Engstrom, Q. Yang, N. Menad, M. Lidstrom y B. Bjorkman, "Characteristics of steel slag under different cooling conditions," Waste Management, vol. 27, pp. 1335-1344, 2007. DOI: 10.1016/j.wasman.2006.08.002.

[74] B.V. Kök y N. Kuloğlu, "Effects of steel slag usage as aggregate on indirect tensile and creep modulus of hot mix asphalt," Gazi University Journal of Sience, vol. 21, N. 3, pp. 97-103, 2008.

[75] G. Wang, "Determination of the expansion force of coarse steel slag aggregate," Construction and Building Materials, vol. 24, pp. 1961-1966, 2010. DOI: 10.1016/j.conbuildmat.2010.04.004.

[76] M. Ameri, S. Hesami y H. Goli, "Laboratory evaluation of warm mix asphalt mixtures containing electric arc furnace (EAF) steel slag," Construction and Building Materials, vol. 49, pp. 611-617, 2013. DOI: 10.1016/j.conbuildmat.2013.08.034.

[77] J. Xie, J. Chen, S. Wu, J. Lin y W. Wei, "Performance characteristics of asphalt mixture with basic oxygen furnace slag," Construction and Building Materials, vol. 38, pp. 796-803, 2013. DOI: 10.1016/j.conbuildmat.2012.09.056.

[78] G.H. Shafabakhsh y O.J. Ani, "Experimental investigation of effect of nano $\mathrm{TiO} 2 / \mathrm{SiO} 2$ modified bitumen on the rutting and fatigue performance of asphalt mixtures containing steel slag aggregates," Construction and Building Materials, vol. 98, pp. 692-702, 2015. DOI: 10.1016/j.conbuildmat.2015.08.083.

[79] G.H. Shafabakhsh, O.J. Ani y M. Talebsafa, "Artificial neural network modeling (ANN) for predicting rutting performance of nano-modified hot-mix asphalt mixtures containing steel slag aggregates," Construction and Building Materials, vol. 85, pp. 136-143, 2015a. DOI: 10.1016/j.conbuildmat.2015.03.060.

[80] L. Hunt y G. Boyle, "Steel slag in hot mix asphalt concrete," Final Report, State Research Project \#511, Oregon Department of Transportation, 2000.

[81] D.H. Shen, C.M. Wu y J.C. Du, "Laboratory investigation of basic oxygen furnace slag for substitution of aggregate in porous asphalt mixture," Construction and Building Materials. vol. 23, pp. 453-461, 2009. DOI: 10.1016/j.conbuildmat.2007.11.001.

[82] Y. Xue, H. Hou, S. Zhu y J. Zha, "Utilization of municipal solid waste incineration ash in stone mastic asphalt mixture: pavement performance and environmental impact," Construction and Building Materials, vol. 23, N. ${ }^{\circ}$, pp.989-96, 2009. DOI: 10.1016/j.conbuildmat.2008.05.009. 
[83] J. Xie, Z. Chen, L. Pang y S. Wu, "Implementation of modified pull-off test by UTM to investigate bonding characteristics of bitumen and basic oxygen furnace slag (BOF)," Construction and Building Materials, vol. 57, pp. 61-68, 2014. DOI: 10.1016/j.conbuildmat.2014.01.083.

[84] JEGEL. "Steel slag aggregates use in hot mix asphalt concrete," Final report prepared for the steelmaking slag Technical Committee, Toronto, John Emery Geotechnical Engineering Limited (JEGEL), 1993.

[85] J. Emery, "Steel slag utilization in asphalt mixes," National Slag Association MF 186-1. Canadian Technical Asphalt Association Proceedings, 1986, 11 p.

[86] W.T. Kuo y C.Y. Shu. "Application of high-temperature rapid catalytic technology to forecast the Volumetric stability behavior of containing steel slag mixtures," Construction and Building Materials, vol. 50, pp. 463-470, 2014. DOI: 10.1016/j.conbuildmat.2013.09.030.

[87] Q. Li, H. Ding, A. Rahman y D. He, "Evaluation of Basic Oxygen Furnace (BOF) material into slag-based asphalt concrete to be used in railway substructure". Construction and Building Materials, vol. 115, pp. 593-601, 2016. DOI: 10.1016/j.conbuildmat.2016.04.085.

[88] M. Ameri y B. Behnood, "Laboratory studies to investigate the properties of CIR Mixes containing steel slag as a substitute for virgin aggregates," Journal of Construction and Building Materials, vol. 26, N. ${ }^{\circ}$ 1, pp. 475-480, 2012. DOI: 10.1016/j.conbuildmat.2011.06.047.

[89] Z. Chen, S. Wu, J. Wen, M. Zhao, M. Yi y J. Wan, "Utilization of gneiss coarse aggregate and steel slag fine aggregate in asphalt mixture," Construction and Building Materials, vol. 93, pp. 911-918, 2015. DOI: 10.1016/j.conbuildmat.2015.05.070.

[90] A. Hiltunen, "The influence of chemical and physical properties on the utilization of slags," presentado en Sixth International Conference on Molten Slags, Fluxes and Salts, Finland, 2000 .

[91] B.J. Reeves y W.K. Lu, "High temperature modification of steelmaking slags by the addition of fayalite slag to react a Volumetrically stable aggregate," Presentado en Sixth International Conference on Molten Slags, Fluxes and Salts, Finland, 2000.

[92] Y. Shi, H. Chen, J. Wang y Q. Feng, "Preliminary investigation on the pozzolanic activity of superfine steel slag," Construction and Building Materials, vol. 82, pp. 227-234, 2015.

[93] M. Tiifekqi, A. Demirbas y H. Genc, "Evaluation of steel furnace slags as cement additives," Cement and Concrete Research. vol. 27, N. ${ }^{\circ} 11$, pp. 1713-1717, 1997. DOI: 10.1016/ S0008-8846(97)00158-0.

[94] H. Wen, E. Wu y S. Bhusal, "Evaluation of steel slag as hot mix asphalt aggregate," Final report, Edw. C. Levy Co. and Nucor Steel Seattle, Inc., Seattle, WA, 2014.

[95] H. Ziari, y M.M. Khabiri, "Preventive maintenance of flexible pavement and mechanical properties of steel slag asphalt," Journal of Environmental Engineering and Landscape Management, vol. 15, N. ${ }^{\circ}$ 3, pp. 188b-192b., 2007. DOI: 10.1080/16486897.2007.9636928.

[96] .A. Oluwasola, M.R. Hainin, M.M.A. Aziz, H. Yaacob y M.N.M. Warid, "Potentials of steel slag and copper mine tailings as construction materials," Materials Research Innovations, vol. 18, S6, pp. 250-254, 2014. DOI: 10.1179/1432891714Z.000000000966. 
[97] H. Wen, S. Wu y S. Bhusal, "Performance evaluation of asphalt mixes containing steel slag aggregate as a measure to resist studded tire wear," Journal of Materials in Civil Engineering, vol. 28, N. ${ }^{\circ}$ 5, 2016. DOI: 10.1061/(ASCE)MT.1943-5533.0001475.

[98] U. Bagampadde, H.I.A. Wahhab y S.A. Aiban, "Optimization of steel slag aggregates for bituminous mixes in Saudi Arabia," Journal of Materials in Civil Engineering, vol. 11, N. ${ }^{\circ}$ 1, pp. 30-35, 1999. DOI: 10.1061/(ASCE)0899-1561(1999)11:1(30).

[99] L.S. Huang, D.F. Lin, H.L. Luo y P.C. Lin, "Effect of field compaction mode on asphalt mixture concrete with basic oxygen furnace slag," Construction and Building Materials, vol. 34, pp. 16-27, 2012. DOI: 10.1016/j.conbuildmat.2012.02.008.

[100] S.-H. Chen, J.-D. Lin, D. Huang y C.-T. Hung, "Performance of replacing traditional natural aggregates in dense grade asphalt concrete with basic oxygen furnace slag," presentado en Geo-Hubei 2014 International Conference on Sustainable Civil Infrastructure, China, pp. 107-114, 2014. DOI: 10.1061/9780784478554.014.

[101] E.A. Oluwasola, M.R. Hainin y M.M.A. Aziz, "Evaluation of asphalt mixtures incorporating electric arc furnace steel slag and copper mine tailings for road construction," Transportation Geotechnics, vol. 2, pp. 47-55, 2015. DOI: 10.1016/j.trgeo.2014.09.004.

[102] Z. Chen, J. Xie, Y. Xiao, J. Chen y S. Wu, "Characteristics of bonding behavior between basic oxygen furnace slag and asphalt binder," Construction and Building Materials, vol. 64, pp. 60-66, 2014. DOI: 10.1016/j.conbuildmat.2014.04.074.

[103] I. Liapis y S. Likoydis, "Use of electric arc furnace slag in thin skid-resistant surfacing," Procedia -Social and Behavioral Sciences, vol. 48, pp. 907-918, 2012. DOI: 10.1016/j. sbspro.2012.06.1068.

[104] I.S. Bessa, V. Castelo-Branco y J. Barbosa-Soares, "Evaluation of polishing and degradation resistance of natural aggregates and steel slag using the aggregate image measurement system," Road Materials and Pavement Design, vol. 15, N. ${ }^{\circ} 2$, pp. 385-405, 2014. DOI: 10.1080/14680629.2014.883323.

[105] Z. Chen, S. Wu, Y. Xiao, M. Zhao y J. Xie, "Feasibility study of BOF slag containing honeycomb particles in asphalt mixture," Construction and Building Materials, vol. 124, pp. 550-557, 2016. DOI: 10.1016/j.conbuildmat.2016.07.128.

[106] L.S. Huang, G.L. Zou, H.L. Luo y C.C. Chao, "In-situ temperature effects in basic oxygen furnace slag asphalt concrete pavement," International Journal of Pavement Research and Technology, vol. 6, N. 4, pp. 386-94, 2013.

[107] D.F. Lin, L.H. Chou, Y.K. Wanga y H.L. Luo, "Performance evaluation of asphalt concrete test road partially paved with industrial waste -Basic oxygen furnace slag," Construction and Building Materials, vol. 78, pp. 315-323, 2015. DOI: 10.1016/j.conbuildmat.2014.12.078.

[108] Y. Xue, H. Hou y S. Zhu, "Adsorption removal of reactive dyes from aqueous solution by modified basic oxygen furnace slag: isotherm and kinetic study," Chemical Engineering Journal, vol. 147, N. ${ }^{\circ} 2-3$, pp. 272-279, 2009a. DOI: 10.1016/j.cej.2008.07.017.

[109] Y.C. Hsu, "The evaluation of temperature on asphalt concrete with basic oxygen furnace slag mixed," Tesis de maestría, Ingeniería, I-Shou University, Taiwan, 2009. 
[110] N.A. Ali, J.S. Chan, T. Papagiannakis, E.G. Theriault y A.T. Bergan, "The use of steel slag in asphaltic concrete,” ASTM Special Technical Publication, pp. 3-18, 1992.

[111] C. Li, Z. Chen, S. Wu, B. Li, J. Xie y Y. Xiao, "Effects of steel slag fillers on the rheological properties of asphalt mastic," Construction and Building Materials, vol. 145, N. ${ }^{\circ}$, pp. 383-391. 2017. DOI: 10.1016/j.conbuildmat.2017.04.034.

[112] D. Topini, E. Toraldo, L. Andena y E. Mariani, "Use of recycled fillers in bituminous mixtures for road pavements," Construction and Building Materials, vol. 159, pp. 189-197, 2018. DOI: 10.1016/j.conbuildmat.2017.10.105. 